\title{
PRIMER REPORTE DE Spodoptera eridania (Stoll) (Lepidoptera: Noctuidae) EN EL CENTRO DE LA PROVINCIA DE SANTA FE
}

\author{
LutZ, A. L. ${ }^{1,2} ;$ BertolaccinI, I. ${ }^{1} ;$ SCOTTA, R. R. ${ }^{1}$ MAntiCA, F. ${ }^{1}$ \\ Magliano, M. F. ${ }^{1} ;$ Sanchez, P. D. ${ }^{1}$ \& Curis, M. C. ${ }^{1}$
}

\begin{abstract}
RESUMEN
Este trabajo reporta por primera vez la presencia de Spodoptera eridania (Stoll) en el centro de la Provincia de Santa Fe. Larvas del género Spodoptera fueron recolectadas en un lote de soja Bt y trasladadas a la cámara de cría (FCA-UNL), donde se obtuvo la primera generación. Mediante claves taxonómicas se corroboró que la especie recolectada era S. eridania, plaga de origen tropical presente en el noroeste de Argentina, de hábito polífago que ocasiona daños al alimentarse de hojas y frutos en formación en varios cultivos de importancia económica. Además esta especie es tolerante a la proteína Bt y resistente a insecticidas de uso convencional. Al alimentar las larvas con hojas de soja Bt, completaron su ciclo biológico de manera exitosa. No existe información acerca del comportamiento de esta especie en el centro de la provincia de Santa Fe.
\end{abstract}

Palabras clave: Glycine max, daños, Oruga militar del sur, Argentina.

\begin{abstract}
First report of Spodoptera eridania (Stoll) in the center of the Santa Fe province.

This work reports for the first time the presence of Spodoptera eridania (Stoll) in the central area of the Santa Fe province. Spodoptera larvae were collected from Bt soybean fields and were transferred to growth chambers (FCA-UNL), where the first generation was obtained. By larvae morphological analysis was confirmed that the species collected was S. erida-
\end{abstract}

1.- Departamento de Producción Vegetal. Facultad de Ciencias Agrarias, Universidad Nacional del Litoral. Kreder 2805. (3080) Esperanza, provincia de Santa Fe, Argentina.

Email: alutz@fca.unl.edu.ar

2.- Consejo Nacional de Investigaciones Científicas y Tecnológicas (CONICET). Becaria doctoral.

Manuscrito recibido el 9 de septiembre de 2019 y aceptado para su publicación el 23 de octubre de 2019.

Lutz, A. L.; Bertolaccini, I.; Scotta, R. R.; Magliano, M. F.; Sánchez, P. D.; Curis, M. C. Primer reporte de Spodoptera eridania (Stoll) (Lepidoptera: Noctuidae) en el centro de la provincia de Santa Fe. FAVE - Ciencias Agrarias 18 (1): 19-24. CC BY-NC-SA 4.0 (c)(1) (2) 


\section{A. L. Lutz et al.}

nia, pest of tropical origin and present in Argentina northwest. Is a polyphagous defoliator that feeds on several species of economic importance, with a high tolerance to the Cry1 Ac protein, which is expressed in some Bt crops and is resistant to conventional insecticides. When the larvae were fed with Bt soybeans, they successfully completed their biological cycle. There is not information about the behavior of this species in the central area of the Santa Fe province.

Key words: Glycine max, damage, Southern armyworm, Argentina.

La soja (Glycine max (L.) Merrill) es el cultivo agrícola de mayor importancia en Argentina, con una superficie sembrada de 17,3 millones de hectáreas y una producción de 56,5 millones de toneladas durante la campaña agrícola 2018/2019 (4), siendo la principal área de producción el centro del país (21).

Los insectos defoliadores constituyen una de las plagas de mayor importancia en el cultivo de soja, ocasionando una disminución del área foliar que compromete notablemente el rendimiento. Entre las especies de mayor frecuencia de aparición y abundancia en la zona central de la Provincia de Santa Fe se encuentran Anticarsia gemmatalis (Hübner) y Rachiplusia $n u$ (Guenée) $(2,12,14,25)$. Sin embargo, luego de la incorporación al mercado de cultivares de soja genéticamente modificada que expresan un gen de Bacillus thuringiensis Berliner con actividad insecticida sobre lepidópteros (soja Bt) (6), se observó una modificación del hábitat dado por la aparición de otras especies plaga sobre las cuales la tecnología Bt no ejerce control como por ejemplo las orugas del género Spodoptera $(19,27)$. Tal es el caso de Spodoptera cosmiodes (Walker) y Spodoptera eridania (Stoll), ambas con tolerancia a la proteína insecticida Cry1Ac que expresan estos cultivares y resistentes a varios insecticidas $(3,6,16)$. En la provincia de Santa
Fe ya se ha registrado la presencia de $S$. cosmioides atacando cultivos de soja convencional y $\mathrm{Bt}(13,15)$. Sin embargo, para S. eridania y según SINAVIMO (Sistema Nacional Argentino de Vigilancia y Monitoreo de Plagas, Servicio Nacional de Sanidad y Calidad Agroalimentaria) sólo existe registro de su presencia en la región noroeste del país $(1,26)$.

Spodoptera eridania, comunmente llamada "oruga militar del sur", es una especie nativa de los trópicos americanos (20). Es un lepidóptero polífago que causa daño al alimentarse de hojas y frutos en formación en numerosos cultivos agrícolas, frutales y ornamentales $(5,8,9,11,16,23)$. Además, algunas malezas se comportan como hospederos alternativos de esta especie (22), demostrando la adaptabilidad y plasticidad alimentaria que tiene esta plaga a diferentes agroecosistemas (6).

Durante monitoreos de plagas insectiles realizados en cultivos de soja Bt en la zona centro de la provincia de Santa Fe, cercanos a la localidad de Santa María Norte $\left(31^{\circ} 31^{\prime} 00^{\prime \prime} \mathrm{S} 61^{\circ} 08^{\prime} 00^{\prime \prime} \mathrm{O}\right)$, durante la campaña 2018/2019, se detectó la presencia de larvas pertenecientes al género Spodoptera en una densidad poblacional elevada (15 larvas por metro lineal) (Figura 1.A). Se realizó la colecta de estos individuos y posterior traslado a la cámara de cría, hasta lograr la primera generación en condiciones 
controladas. Las larvas fueron identificadas siguiendo las claves taxonómicas de Oliver (1981) (18), Caballero et al (1994) (7) y del boletín OEPP/EPPO (2015) (17) mediante las cuales se corroboró que la especie recolectada era S. eridania.

Si bien pertenecen al mismo género, $S$. eridania y $S$. cosmiodes presentan aspectos característicos que permiten su diferenciación. Al igual que S. cosmiodes, los huevos de $S$. eridania son colocados en masa, sin embargo, en la "oruga militar del sur" presentan coloración verdosa (24) (Figura 1.B). Durante el estado larval exhibe un ensanchamiento del último segmento torácico y primero abdominal ("giba"), rasgo que comparte con S. cosmiodes, sin embargo, ambas especies se diferencian en la banda sub-espiracular que recorre el abdomen y tórax: en $S$. cosmiodes es continua, mien- tras que en S. eridania se interrumpe y continúa en forma de línea suave en el tórax (10) (Figura 1.C).

Para conocer más acerca de la biología de S. eridania se procedió a evaluar algunos parámetros biológicos de la especie. Para ello se realizó la cría de los ejemplares recolectados a campo en condiciones controladas de temperatura $\left(25^{\circ} \mathrm{C}\right)$, humedad relativa $(60 \%)$ y fotoperiodo $(14: 10 \mathrm{hs}$ de luz:oscuridad). Las larvas neonatas, fueron criadas individualmente en cajas de Petri y se alimentaron con hojas frescas de soja Bt, cultivada en el campo experimental de la FCA. Se registró la duración promedio del estado larval (28,11 $\pm 3,16$ días), del estado pupal (12 $\pm 1,22$ días) y del estado adulto $(9,74 \pm 2,08$ días $)$, siendo estos valores similares a los hallados por Góméz López et al. (2009) (11) y Bortolotto et al (2014)
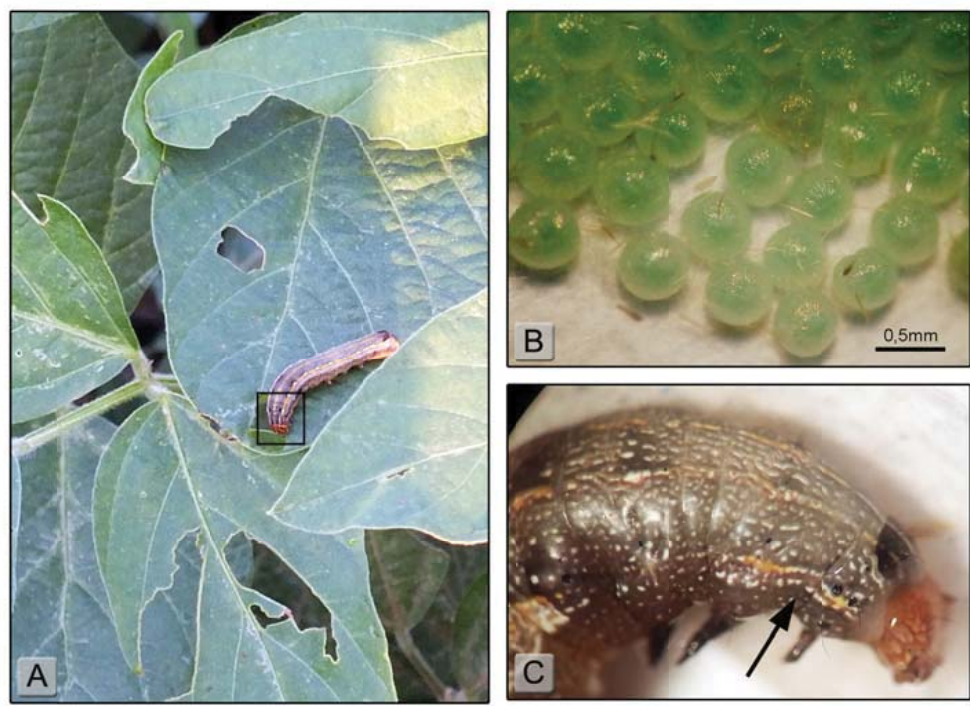

Figura 1. Spodoptera eridania (Stoll) defoliando hojas de soja Bt (A); Detalle de la masa de huevos, de coloración verdosa característica (B); Banda sub-espiracular discontinua, rasgo propio de S. eridania (C). Fotos: A. L. 
(6) en poblaciones de S. eridania alimentadas con soja convencional y $\mathrm{Bt}$, respectivamente.

Este trabajo constituye el primer reporte de S. eridania atacando el cultivo de soja en el centro de la provincia de Santa Fe. Además, debido al incremento de la superficie cultivada con variedades que expresan las proteínas de acción insecticida "Cry", de las cuales esta especie es tolerante (3), y al daño significativo que pueden ocasionar en el cultivo al alimentarse de hojas y vainas en formación, es una plaga que requiere de un estado de alerta permanente sobre su dinámica poblacional. Futuros trabajos de investigación son necesarios para conocer el comportamiento de la S. eridania en condiciones de campo, aspecto importante para lograr la implementación de un manejo integrado exitoso.

\section{AGRADECIMIENTOS}

A la Ing. Agr. Laura Fernández por la edición de las imágenes y al Ing. Agr. José Menegón, propietario del lote donde se recolectaron las larvas de S. eridania.

Los recursos para el presente trabajo fueron aportados por la Universidad Nacional del Litoral a través del programa $\mathrm{CAI}+\mathrm{D}$ (No 50120150100131LI).

\section{BIBLIOGRAFÍA}

1.- ACOSTA, L.G; BARRIONUEVO, M.J. Y CHALUP, A.E. 2015. Identificación de dos estadios larvales de cuatro especies de Spodoptera Guenée (Lepidoptera: Noctuidae) presentes en cultivos de soja (Glycine max) en el Noroeste Argentino. XI Congreso Argentino de Entomología. Posadas; Misiones.

2.- ARAGÓN, J.R. 2002. Insectos Perjudiciales de la Soja. Instituto Nacional de Tecnología Agropecuaria. EEA Marcos Juárez. Revista de Información sobre Investigación y Desarrollo Agropecuario (IDIA XXl). Oleaginosas, Sección Soja. $N^{\circ}$ 3, 75-82.

3.- BERNARDI, O.; SORGATTO, R.; BARBOSA, A.; DOMINGUES, F.; DOURADO, P.; CARVHALO, R.; MARTINELLI, S.; HEAD, G. Y OMOTO, C. 2014. Low susceptibility of Spodoptera cosmioides, Spodoptera eridania and Spodoptera frugiperda (Lepidoptera: Noctuidae) to genetically modified soybean expressing Cry1Ac protein. Crop Protection 58: 33-40.

4.- BOLSA DE CEREALES DE ROSARIO. 2019. Guía estratégica para el agro. Estimaciones de producción. Disponible en: https://bcr.com.ar/es/ mercados/gea. Acceso: 25/7/2019.

5.- BORTOLI, S.A.; MURATA, A.T.; VACARI, A.M.; BORTOLI, C.P. Y RAMALHO, D.G. 2012. Herbivoriaem soja: efeito na composic, ão química das folhas e na biologia da lagarta da sojae do percevejo verde pequeno. Comunicata Scientiae 3: 192-198.

6.- BORTOLOTTO, O.C.; SILVA, G.V.; DE FREITAS BUENO, A.; POMARI, A.F.; MARTINELLI, S. HEAD, G.P.; CARVALHO, R.A. Y BARBOSA, G.C. 2014. Development and reproduction of Spodoptera eridania (Lepidoptera: Noctuidae) and its egg parasitoid Telenomus remus (Hymenoptera: Platygastridae) on

Revista FAVE - Ciencias Agrarias 18 (2) 2019 
the genetically modified soybean (Bt) MON $87701 \times$ MON 89788. Bulletin of Entomological Research 104: 724-730.

7.- CABALLERO, R.; HABECK, D.H. Y ANDREWS, K.L. 1994. Clave ilustrada para larvas de Noctuídos de importância econômica de El Salvador, Honduras y Nicarágua. CEIBA 35(2): 2237-2252.

8.- DELANEY, K.J. 2012. Nerium oleander indirect leaf photosynthesis and light harvesting reductions after clipping injury or Spodoptera eridania herbivory: high sensitivity to injury. Plant Science 185-186: 218-226.

9.- FAVETTI, B.M.; BUTNARIUB, A.R. Y FOERSTERC, L.A. 2015. Biology and reproductive capacity of Spodoptera eridania (Cramer) (Lepidoptera, Noctuidae) in different soybean cultivars. Revista Brasileira de Entomologia 59: 89-95.

10.- FICHETTI, P.; NAVARRO, F. Y MAS, G. 2010. Identificación de orugas en soja. Capítulo 3. En: Igárzabal, D.; Fichetti, P.; Navarro, F.; Mas, G. y Morre, J. 2010. Manejo de orugas defoliadoras. L.I.D.E.R. Laboratorio de Investigación Desarrollo y Experimentación Regional. Córdoba. Edición Dupont. 95p. 11.- GÓMEZ LÓPEZ, V.A.; CABRAL ANTUNEZ, C.C. Y RAMIREZ DE LÓPEZ, M.B. 2009. Aspectos biológicos de Spodoptera eridania (Lepidoptera; Noctuidae) criadas en diferentes tipos de dieta. Plaga del cultivo de soja. San Lorenzo, Paraguay: Dpto. De Proyección Vegetal, FCA-UNA/INBIO. 40 p. Disponible en: http://www.agr.una.py/ fca/index.php/libros/catalog/view/4/3/3341 (Acceso: 30 de Julio de 2019).

12.- IGARZÁBAL, D.A.; GALVEZ, M.C.; ALDREY, M.C.; PERALTA, C.R.; CACCIAVILLANI, J.I. Y GASSEN, D.N. 2015. Orugas y chinches en soja. Universidad Católica de Córdoba. 271 p.
13.- LUTZ,A.; SCOTTA, R.; CURIS, C.; BERTOLACCINI, I.; ROSETTI, L. Y DOMIZI, L. 2015. Efecto de la soja Bt en la población de artrópodos plagas y benéficos en el Centro de la Provincia de Santa Fe. XV Jornadas Fitosanitarias Argentinas. Santa Fe, Argentina. 14.- MASSARO, R. 2008. Plagas insectiles del cultivo de soja. En: Fernández Alsina, M. Para mejorar la producción. Publicaciones Regionales Instituto Nacional de Tecnología Agropecuaria. EEA Oliveros (45): 95-101.

15.- MASSONI F.A.; SCHLIE, G. Y FRANA, J. 2014. Cultivo de soja Bt (RR2 PRO) y convencional (RR1) expuestos a poblaciones naturales de organismos plaga y depredadores. Instituto Nacional de Tecnología Agropecuaria (INTA). EEA Rafaela. Información técnica de cultivos de verano. Campaña 2013/2014. Publicación Miscelánea 128: 82-89.

16.- MONTEZANO, D.G.; SPECHT, A.; SOSA-GÓMEZ, D.R.; ROQUE-SPECHT, V.F. Y DE BARROS, N.M. 2014. Immature stages of Spodoptera eridania (Lepidoptera: Noctuidae): developmental parameters and host plants. Journal of Insect Science 14: 1-11. 17.- OEPP/EPPO. 2015. Bulletin PM 7/124. Spodoptera littoralis, Spodoptera litura, Spodoptera frugiperda, Spodoptera eridania. European and Mediterranean Plant Protection Organization. Organisation Européenne et Méditerranéenne pour la Protection des Plantes 45: 410-444. 18.- OLIVER, A. D. 1981. Biology and illustrated key for the identification of twenty species of economically important noctuid pests. LSU Agricultural Experiment Station Reports. 260 p. 
19.- PEROTTI, E.; BOERO, L.; TAMAG- 24.- TEODORO, A.V.; PROCÓPIO, S.O.; NONE, M.; MACCARI, G.; ESCUDE, B. BUENO, A.F.; NEGRISOLI JR, A.S.; Y GAMUNDI, J. 2016. Evaluación de tres es- CARVALHO, H.W.L.; NEGRISOLI, trategias de manejo del complejo de plagas del cultivo desoja. Instituto Nacional deTecnología Agropecuaria (INTA). EEA Oliveros. 8 p.

20.- POGUE, G.M. 2002. A world revision of the genus Spodoptera Gueneé (Lepidoptera: Noctuidae). Memoirs of the American Entomological Society 43: 1-202.

21.- ROCHA, P. Y VILLALOBOS, V.M. 2012. Estudio comparativo entre el cultivo de soja genéticamente modificada y el convencional en Argentina, Brasil, Paraguay y Uruguay. Ministerio de Agricultura, Ganadería y Pesca, Argentina. Instituto Interamericano de Cooperación para la Agricultura, Costa Rica. 90 p.

22.- SANTOS, K. B.; MENEGUIM, A. M. Y NEVES, P.M.O.J. 2005. Biologia de Spodoptera eridania (Cramer) (Lepidoptera: Noctuidae) em diferentes hospedeiros. Neotropical Entomology 34: 903-910.

23.- SILVA, A.; BARONIO, C.A.; GALZER, E.C. W. GARCIA, M.S. Y BOTTON, M. 2019. Development and reprotuction of Spodoptera eridania on natural hosts and artificial diet. Brazilian Journal of Biology 79 (1): 80-86.
C.R.C.B.; BRITO, L.F. Y GUZZO, E.C. 2013. Spodoptera cosmioides (Walker) e Spodoptera eridania (Cramer) (Lepidoptera: Noctuidae): novas pragas de Cultivos na Região Nordeste. Aracajú. Embrapa Tabuleiros Costeiros.Comunicado Técnico 131.7p.

25.- URRETABIZKAYA, N.; VASICEK, A. Y SAINI, E. 2010. Insectos perjudiciales de importancia agronómica. 1: Lepidópteros. Ediciones Instituto Nacional de Tecnología Agropecuaria (INTA). 77 p. 26.- VALVERDE, L.; ROMERO SUELDO, M.; COLOMO, M.V.; BERTA, C. Y DODE, M. 2008. Lepidópteros noctuidos, plagas en el cultivo de soja en Tucumán, Argentina. Boletín Sanidad Vegetal Plagas 34 (3): 377-381.

27.- ZHAO, J.H.; HO, P. Y AZADI, H. 2011. Benefits of $\mathrm{Bt}$ cotton counter balanced by secondary pests? Perceptions of ecological change in China. Environmental Monitoring and Assessment 173: 985-994. 